\title{
Temperature Distribution Monitoring Using Ultrasonic Thermometry Based on Markov Radial Basis Function Approximation and Singular Values Decomposition
}

\author{
Xuehua Shen,, Qingyu Xiong, ${ }^{2,3}$ Weiren Shi, ${ }^{1}$ Kai Wang, ${ }^{1}$ and Guoqiang Lai ${ }^{1}$ \\ ${ }^{1}$ School of Automation, Chongqing University, Chongqing 400044, China \\ ${ }^{2}$ Key Laboratory of Dependable Service Computing in Cyber Physical Society, MOE, Chongqing 400044, China \\ ${ }^{3}$ School of Software Engineering, Chongqing University, Chongqing 400044, China \\ Correspondence should be addressed to Qingyu Xiong; cquxqy@163.com
}

Received 26 December 2013; Revised 24 March 2014; Accepted 2 April 2014; Published 28 April 2014

Academic Editor: Ping-Lang Yen

Copyright ( 2014 Xuehua Shen et al. This is an open access article distributed under the Creative Commons Attribution License, which permits unrestricted use, distribution, and reproduction in any medium, provided the original work is properly cited.

\begin{abstract}
Information about temperature distribution is complex but of critical importance for the control of various microwave applications. In this paper, an innovative way of temperature distribution monitoring using ultrasonic thermometry in microwave field is investigated. The principle of ultrasonic thermometry in the situation of ideal gas is elaborated, and reconstruction algorithm based on Markov radial basis function approximation and singular values decomposition is presented and described in detail. In order to validate the performance of temperature distribution reconstruction of our presented algorithm, four two-dimensional temperature distribution models with different complexities are utilized in simulation experiments. Especially, simulation experiments taking error of measurement into account are studied to verify the robustness. Figure profiles show remarkable correspondence between the reconstructed ones and their models, while quantitative analysis, including the overall temperature error analysis and the hotspot positioning analysis, shows that different kinds of errors calculated are all within the limit ranges. In addition, the time analysis of simulation experiments also demonstrates its well real-time capability.
\end{abstract}

\section{Introduction}

As a novel energy source, microwave heating mode has been applied widely in many industrial fields, including chemical engineering, biodiesel reaction, and material processing. The advantages of microwave applications lie in energy saving, reduction in process duration, quality improving, instantaneous control, and many other unique characteristics induced by noncontact "volumetric" energy absorption [1-7]. However, there are some serious problems, such as inhomogeneous heating, thermal runaway, and transient behavior, which may lead to severe accidents like explosions or spontaneous ignitions [8-14]. These undesired phenomena are due to the difference of heating mode between microwave and other traditional thermal transmissions [4, 12-15]. The traditional thermal transmission mode heats the objects gradually from their surface to the inside. But the microwave works by exciting molecules of the objects, and temperature rises faster from the inside to the surface. In addition, the states of most chemical or physical reactions under microwave processing can often be embodied by the temperature distribution. For example, the effective permittivity of the mixed reactant exhibits a strong dependence on the temperature, and it can be beneficial for the reaction if the temperature can be gained and controlled $[16,17]$. So it is very important for microwave applications to show the inside temperature distribution of the objects.

Common mercury thermometers cannot be used for temperature measurement in the microwave field because they absorb microwaves, which may lead to a spark discharge and consequently destroy the thermometers [4]. For other conventional measurement techniques, such as thermocouples and thermal resistances, there exist some problems with reliability and accuracy, because temperature measurement can only be accomplished when they reach thermal equilibrium state with the measure area [11, 15, 18-20]. Thus, they 
may not be appropriate for monitoring the transient variation in temperature because of their relatively slow time response in measurement. Besides, the main disadvantage hindering them from being used under microwave environment is that they are susceptible to electromagnetic interference, which may result in temperature rise severely. Also the electromagnetic field may be disturbed by these devices in turn. An infrared radiation technique is conveniently used for measuring temperature on the material surface in a noncontact way [11]. However, temperature is always measured on the wall of the reactor, and influence due to different emissivity and reflectivity of infrared radiations from other sources often results in deterioration of measurement accuracy [11, 19, 21]. The recent popular technique of fiber optics is not suitable for monitoring nonuniform temperature distribution either, because it also requires direct contact with the measure objects, which is the similar way as thermocouples and thermal resistances. Furthermore, it will be accompanied with another drawback of the narrow operating range from 0 to $330^{\circ} \mathrm{C}$, while temperature in microwave field is always much higher $[4,22]$. Additionally, the above techniques have the common defect of only providing measurement for a single point, but not for temperature distribution, while temperature distribution is a much more important factor for the control of microwave applications.

In a word, the common temperature measurement techniques are not appropriate for monitoring the transient temperature distribution in microwave field.

To solve problems mentioned above, we study a new temperature measurement technique by ultrasound or ultrasonic thermometry. It is carried out to be an alternative of temperature distribution monitoring in microwave field. Ultrasonic thermometry is a noncontact or nonintrusive method, as ultrasound has high sensitivity to temperature referred to previous researches. Based on the relationship between the velocity of ultrasound and the properties of the medium, which the ultrasound travels through, the average temperature along its travel path can easily be calculated after measuring the ultrasound velocity between the ultrasonic transmitter and receiver [18, 23-25]. Moreover, when multiple transmitters and receivers are properly installed, temperature distribution over the two- or three-dimensional space is possible to be reconstructed in real time. Thus, one can gain the information of temperature distribution and then analyze and control the microwave processing after measuring ultrasound velocity distribution over the twoor three-dimensional measure area with numerous paths between transmitters and receivers [23-25].

This paper is organized as follows. Section 2 describes the basic principle of ultrasonic thermometry. In Section 3, we elaborate the arrangement of transducers and division of the measure area and then present a reconstruction algorithm based on Markov radial basis function approximation and singular values decomposition. Section 4 utilizes four twodimensional temperature distribution models with different complexities to validate the performance of temperature distribution reconstruction of our presented algorithm and gives experiment results and analysis. Conclusions and future research are presented in Section 5.

\section{The Principle of Ultrasonic Thermometry}

The field of ultrasonic thermometry is of particular interest because of its ability to measure temperature without inserting detective instrumentation into the measure object or measure area. The simplest ultrasonic pyrometer is composed of one ultrasonic transmitter mounted on one side and one ultrasonic receiver mounted on the other side along the same path. The transmitter is used to produce ultrasonic signal at a specific time, and the receiver at the known distance is responsible for detecting the ultrasonic signal.

Temperature determination by ultrasound is based on the temperature dependence of ultrasound velocity or that the ultrasound velocity in any medium is a function of temperature [18-20, 23-25]. Such as in ideal gases, the ultrasound velocity is directly proportional to the square root of the temperature, and in most of the liquids the dependence is linear, while in solid objects the ultrasound velocity generally decreases with the increment of the temperature [20]. All the relationships between the ultrasound velocity and the temperature of the medium which the ultrasound travels through can either be known from the existing research materials or be determined from experiment studies. In this paper, the principle in the situation of ideal gas is given in detail. The relationship between the ultrasound velocity and temperature can be described as the following equation $[18,20]$ :

$$
c=\sqrt{\kappa \frac{R}{m} T}=B \sqrt{T},
$$

where $c$ is the ultrasound velocity, $T$ is the gas absolute temperature, and $R$ is the universal gas constant, while $\kappa$ and $m$ are the ratio and the average molecular weight of the gas mixture, respectively. As $\kappa, m$, and $R$ are fixed constants that can be measured for the specific gas, they may be replaced by a coefficient $B$ which is also a constant.

If the ultrasound velocity and gas properties are known, then the temperature $T$ can be calculated by (2) derived from (1):

$$
T=\frac{c^{2}}{B^{2}} .
$$

As the distance between the ultrasonic transmitter and ultrasonic receiver is fixed and known, then the velocity of ultrasound can be calculated when travel time of the ultrasound is measured. Thus, (2) can be rewritten as

$$
T=\left(\frac{L}{t_{l}}\right)^{2} \frac{1}{B^{2}},
$$

where $L$ is the distance between the ultrasonic transmitter and ultrasonic receiver and $t_{l}$ is the total time that the ultrasound wave is in transit. From (3), the average temperature along the measure path is obtained.

\section{Reconstruction of Temperature Distribution Using Reconstruction Algorithm}

As analyzed before, temperature distribution is always far more important than single point temperature for safety and 


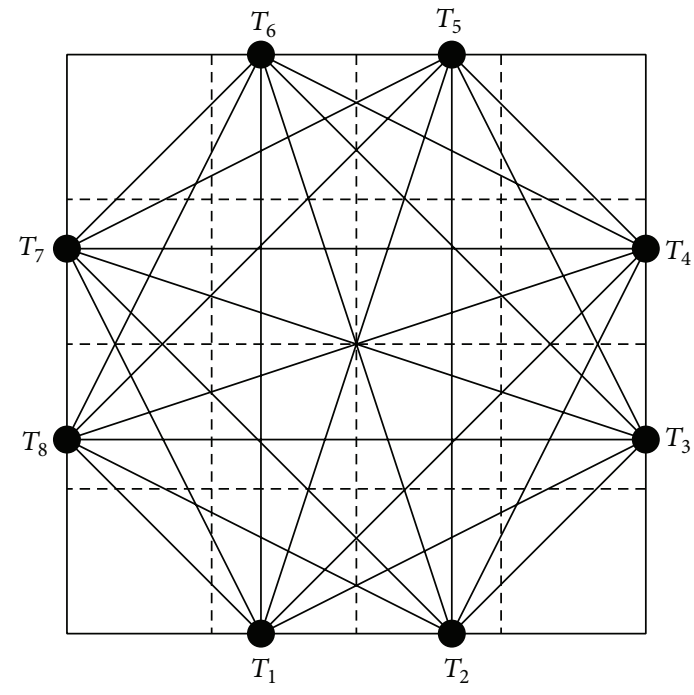

(a)

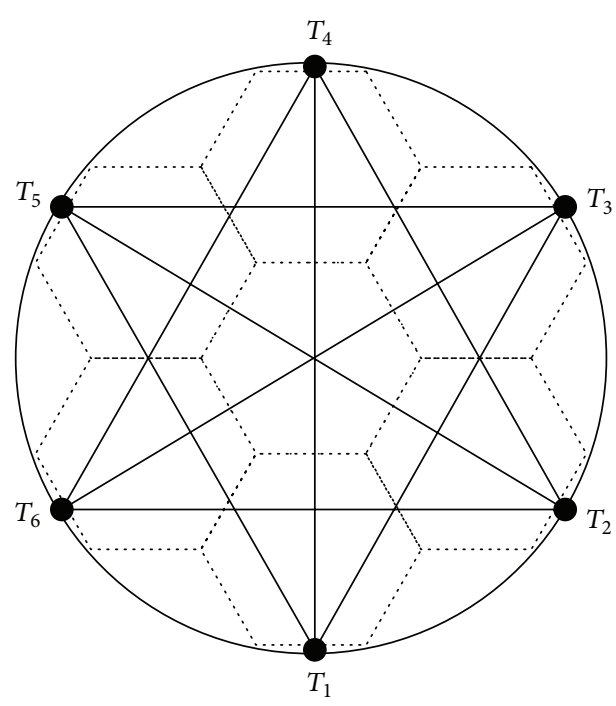

(b)

FIGURE 1: Arrangement of transducers and division of the measure area: (a) square area; (b) circular area.

control in microwave field. However, it may be difficult or even impossible to be obtained via the common methods. Fortunately, reconstruction of temperature distribution over entire measure area will be accomplished using multiple ultrasonic transmitters and receivers, as paths between them can be combined to compute the temperatures of different positions with suitable algorithms.

3.1. Arrangement of Transducers and Division of the Measure Area. The two-dimensional temperature distribution can be gained by installing multiple ultrasonic transmitters and receivers around the perimeter of the measure area to form a multiple paths array, and the measure area may be divided into a number of blocks according to the specific situations; examples can be seen in Figure 1. The ultrasonic transducers are represented by the black symbols, and the effective paths are represented by the solid lines, while the measure area is divided into different amount of blocks by the dashed lines. The ultrasonic transducers used here are called transceivers because of their ability of working as transmitters and receivers, which means they can transmit and detect ultrasonic signal in different time. For example, as shown in Figure 1(a), when transducer $T_{1}$ acts as transmitter and radiates out ultrasonic signal, transducers $T_{2}, T_{3}, \ldots, T_{8}$ play roles of receivers and detect the signal. And then, when transducer $T_{2}$ radiates out ultrasonic signal the other way round, transducers $T_{1}, T_{3}, \ldots, T_{8}$ will detect the signal. Thus and thus, all transducers radiate out the ultrasonic signal in rotation, and other transducers catch the signal from each transducer which serves as transmitter repeatedly.

When all the travel time of these multiple paths has been obtained, then two-dimensional temperature distribution can be reconstructed using suitable algorithms $[24,25]$.

If the transducers are installed over the three-dimensional space, example of cube area is shown as Figure 2; thus the three-dimensional temperature distribution can be obtained

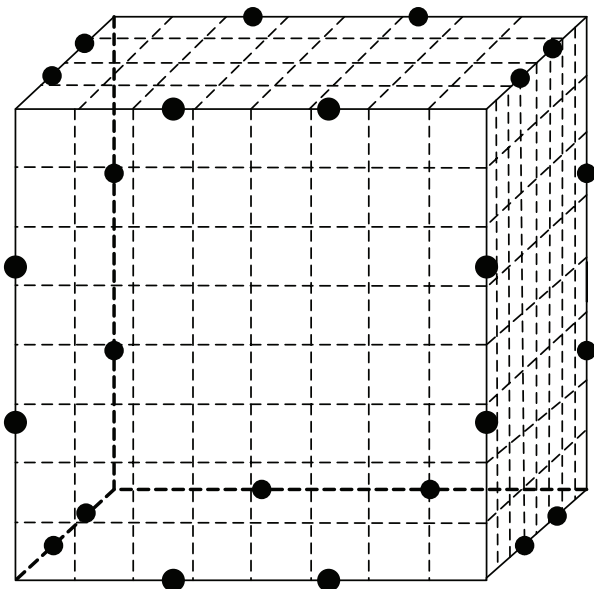

FIGURE 2: Schematic view of transducers installed over the threedimensional space.

by utilizing the data of spatial paths and the reconstruction algorithms applicable to three-dimensional space.

3.2. Reconstruction Algorithm Based on Markov Radial Basis Function Approximation and Singular Values Decomposition. An efficient reconstruction algorithm is the core of temperature distribution monitoring using ultrasonic thermometry. In this paper, reconstruction algorithm based on Markov radial basis function approximation and singular values decomposition is presented and described in detail in the following part.

Assuming that the reciprocal of ultrasound velocity over the measure area is described as function $f(x, y)$, so the travel time of ultrasound wave propagating along a specific path can 
be theoretically expressed as the following equation of line integral:

$$
t_{l_{k}}=\int_{l_{k}} f(x, y) d l_{k}
$$

where $t_{l_{k}}$ is the theoretical value of the travel time of the ultrasound wave and $l_{k}$ is the $k$ th path which the ultrasound wave travels through.

If the measure area is divided into $N$ blocks and $\left(x_{i}, y_{i}\right)$ are the geometric center coordinates of the $i$ th block, then the Markov radial basis function whose center located in $\left(x_{i}, y_{i}\right)$ can be constructed as the following expression:

$$
\phi_{i}(x, y)=e^{-\theta \sqrt{\left(x-x_{i}\right)^{2}+\left(y-y_{i}\right)^{2}}}
$$

where $\theta$ is the shape parameter of the Markov radial basis function, which is related to the specific measure area and the arrangement of the transducers, and it can be predetermined via numerical experiments.

Make the $f(x, y)$ be expressed as a linear combination of the $N$ Markov radial basis functions [26] or given by

$$
f(x, y)=\sum_{i=1}^{N} \varepsilon_{i} \phi_{i}(x, y),
$$

where $\varepsilon_{i}$ is the undetermined coefficient which describes the distribution of the reciprocal of ultrasound velocity over the measure area.

In order to calculate the undetermined coefficient $\varepsilon_{i},(4)$, (5), and (6) are merged as follows:

$$
\begin{aligned}
& t_{l_{k}}=\sum_{i=1}^{N} \varepsilon_{i} \int_{l_{k}} \phi_{i}(x, y) d l_{k}=\sum_{i=1}^{N} a_{k i} \varepsilon_{i}, \\
& a_{k i}=\int_{l_{k}} \phi_{i}(x, y) d l_{k} .
\end{aligned}
$$

If $M$ denotes the amount of the effective paths, now give definitions as

$$
\begin{aligned}
& A=\left(a_{k i}\right), \quad k=1, \ldots, M, i=1, \ldots, N, \\
& \varepsilon=\left(\varepsilon_{1}, \varepsilon_{2}, \ldots, \varepsilon_{N}\right)^{T}, \\
& t=\left(t_{1}, t_{2}, \ldots, t_{M}\right)^{T} .
\end{aligned}
$$

Then (7) can be rewritten as

$$
t=A \varepsilon
$$

Reconstruction of temperature distribution via the travel time of numerous ultrasound paths is an inversion problem, a notable feature of which is the presence of ill-posed problem. Though $A$ is always an ill-conditioned matrix in this inversion problem, effective regularization estimate can be achieved by using the method of singular values decomposition (SVD)
$[27,28]$. Known from analysis based on matrix theory, any real matrix $A \in R^{M \times N}$ can be decomposed as follows:

$$
\begin{aligned}
& A=U S V^{T}, \\
& S=\left[\begin{array}{ll}
\Sigma & 0 \\
0 & 0
\end{array}\right], \quad S \in R^{M \times N}, \\
& \Sigma=\operatorname{diag}\left(\sigma_{1}, \sigma_{2}, \ldots, \sigma_{r}\right),
\end{aligned}
$$

where $U \in R^{M \times M}$ and $V \in R^{N \times N}$ are orthogonal matrices, $\sigma_{1} \geq \sigma_{2} \geq \cdots \geq \sigma_{r}>0$ are the $r$ singular values of matrix $A$, and $r$ is the rank of matrix $A$.

Make the definitions:

$$
\begin{aligned}
& S^{+}=\left[\begin{array}{cc}
\Sigma^{-1} & 0 \\
0 & 0
\end{array}\right], \quad S^{+} \in R^{N \times M}, \\
& \Sigma^{-1}=\operatorname{diag}\left(\frac{1}{\sigma_{1}}, \frac{1}{\sigma_{2}}, \ldots, \frac{1}{\sigma_{r}}\right) .
\end{aligned}
$$

Then the generalized inverse matrix of matrix $A$ or $A^{+} \epsilon$ $R^{N \times M}$ based on singular values decomposition (SVD) can be expressed as

$$
A^{+}=V S^{+} U^{T} \text {. }
$$

Thus, the undetermined coefficient $\varepsilon$ will be expressed as

$$
\varepsilon=A^{+} t .
$$

When the shape of the measure area and the arrangement of all the transducers are known, while the shape parameter $\theta$ is selected appropriately via numerical experiments, then $A^{+}$is predetermined from (12). As $t$ can be obtained by actual measurement, the undetermined coefficient $\varepsilon$ can be calculated from (13). With this, function expression of the reciprocal of ultrasound velocity given by (6) can be gained.

Assuming that the temperature distribution of the measure area is described as function $T(x, y)$, then the temperature distribution of the measure area will be expressed as (14) shown as follows:

$$
T(x, y)=\frac{1}{B^{2} f(x, y)} .
$$

Now, the temperature of any point with known coordinates or the temperature distribution of the fixed measure area can be calculated from (14).

The theory of reconstruction of three-dimensional temperature distribution is basically the same as the situation of two-dimensional temperature distribution, except that the arrangement of the transducers and the reconstruction algorithm are adjusted to be suitable for three-dimensional situation.

The process of temperature distribution reconstruction using ultrasonic thermometry can be summed up by the flow diagram (see Figure 3).

In these applications, temperature distribution monitoring over two-dimensional or three-dimensional space can also detect hotspots which may cause accidents like explosions or spontaneous ignitions, thus contributing to taking protective measures in further control timely. 


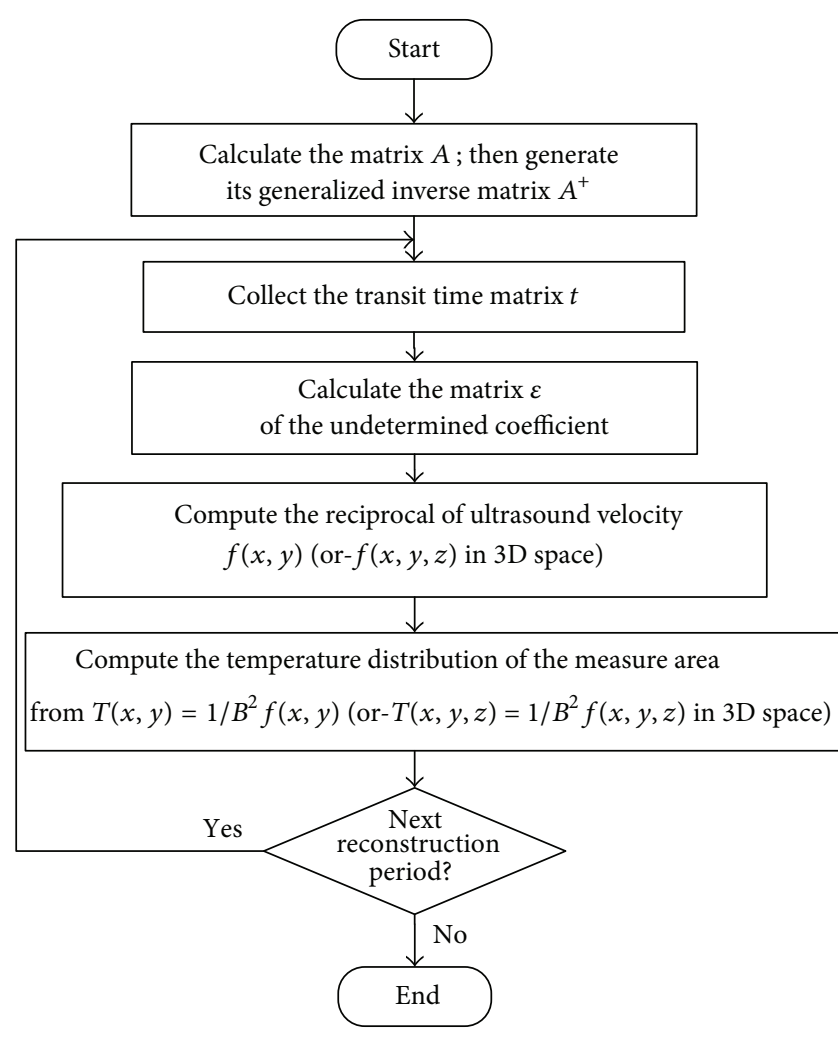

FIgURE 3: Process of temperature distribution reconstruction using ultrasonic thermometry.

\section{Simulation Experiments and Results Analysis}

In order to validate the performance of temperature distribution reconstruction using ultrasonic thermometry based on the algorithm presented in Section 3, simulation experiments in the situation of atmosphere are carried out, and the results analysis is given qualitatively and quantitatively. In this paper, simulation experiments and the results analysis are both done on the MATLAB platform which is a powerful tool for numerical computation, simulation, and visualization.

4.1. Design of Simulation Experiment and Definitions of Evaluation. The reconstruction performance validated here includes feasibility, effectiveness, robustness, and the realtime capability. For a better validation, two groups of simulation experiments in the situation of atmosphere are designed. In the first group of experiments, travel time is assumed to be entirely accurate data which is obtained from theoretical calculation. As that, travel time of the ultrasound waves gained via actual measurement may be different from the theoretical value because of various factors, such as noises or the accuracy limit of measuring devices; the second group of experiments taking error of measurement into account is designed. In these experiments of second group, the travel time of the ultrasound waves is assumed to be overlaid with a Gaussian noise whose mean and standard deviation are 0 and 0.001 , respectively.

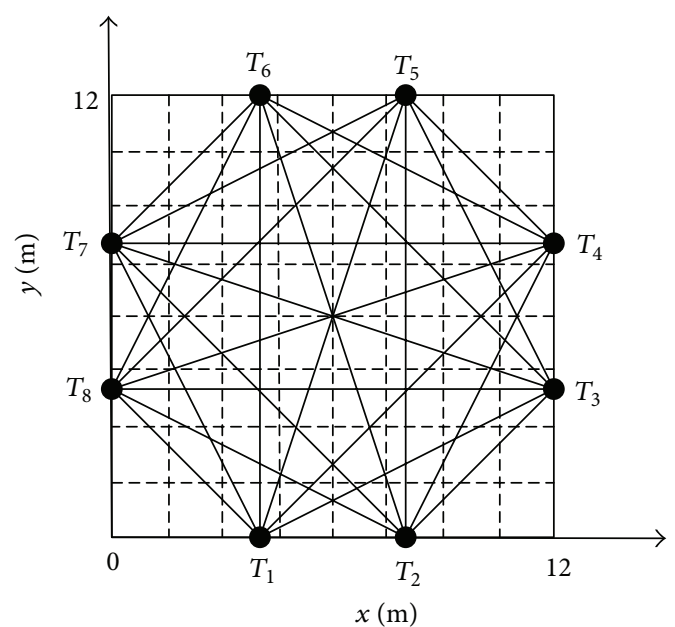

FIGURE 4: Paths array and blocks of measure area in simulation experiments.

Consider an arrangement of 8 ultrasonic transceivers placed around the perimeter of the measure area which is assumed to be square, as shown in Figure 4 . The amount of effective paths represented by the solid lines within the square is 24 , and the measure area is divided into 64 blocks by the dashed lines.

Capability of reconstructing various temperature distributions is important to a reconstruction algorithm of ultrasonic thermometry. In order to validate this capability of our presented algorithm, four kinds of two-dimensional temperature distribution models with different complexity levels are artificially created in MATLAB environment on a computer. Thus, we can gain the travel time arrays of these temperature distribution models via numerical computation and make these models as standard of comparisons when temperature distributions are reconstructed. These twodimensional temperature distribution models created here are shown as the following expressions.

One-peak symmetrical temperature distribution:

$T_{1}(x, y)=800+1000 \times \sin \left(\frac{\pi}{12} x\right) \times \sin \left(\frac{\pi}{12} y\right)$.

One-peak asymmetrical temperature distribution:

$$
T_{2}(x, y)=600+1200 \times e^{\left(-\left((x-8)^{2}+(y-4)^{2}\right) / 100\right)} .
$$

Two-peak asymmetrical temperature distribution:

$$
\begin{aligned}
T_{3}(x, y)= & 800+1000 \times e^{-20\left((x / 12-1 / 3)^{2}+(y / 11-2 / 3)^{2}\right)} \\
& +600 \times e^{-\left(20(x / 12-2 / 3)^{2}+15(y / 11-1 / 3)^{2}\right)} .
\end{aligned}
$$

Three-peak asymmetrical temperature distribution:

$$
\begin{aligned}
T_{4}(x, y)= & 1000 \times e^{-20\left((x / 12-1 / 5)^{2}+(y / 11-5 / 7)^{2}\right)} \\
& +1000 \times e^{-20\left((x / 12-4 / 5)^{2}+(y / 11-4 / 7)^{2}\right)} \\
& +800 \times e^{-\left(20(x / 12-2 / 5)^{2}+15(y / 11-1 / 3)^{2}\right)}+800 .
\end{aligned}
$$


TABLE 1: The overall temperatures error analysis (without noise).

\begin{tabular}{lcc}
\hline & Mean relative error $E_{m}$ & Root-mean-square percent error $E_{r}$ \\
\hline One-peak symmetrical temperature distribution & $4.681 \%$ & $5.335 \%$ \\
One-peak asymmetrical temperature distribution & $1.447 \%$ & $2.126 \%$ \\
Two-peak asymmetrical temperature distribution & $1.318 \%$ & $1.722 \%$ \\
Three-peak asymmetrical temperature distribution & $2.416 \%$ & $3.190 \%$ \\
\hline
\end{tabular}

TABLE 2: The overall temperatures error analysis (with Gaussian noise).

\begin{tabular}{lcc}
\hline & Mean relative error $E_{m}$ & Root-mean-square percent error $E_{r}$ \\
\hline One-peak symmetrical temperature distribution & $4.791 \%$ & $5.409 \%$ \\
One-peak asymmetrical temperature distribution & $1.555 \%$ & $2.175 \%$ \\
Two-peak asymmetrical temperature distribution & $1.729 \%$ & $2.575 \%$ \\
Three-peak asymmetrical temperature distribution & $2.447 \%$ & $3.229 \%$ \\
\hline
\end{tabular}

To evaluate the performance of temperature distribution reconstruction using ultrasonic thermometry based on the presented algorithm, both qualitative and quantitative analyses of reconstruction performance are provided.

The quality of reconstruction performance is evaluated from two aspects: one is the overall temperature error analysis and the other one is the hotspot positioning analysis.

The overall temperature error analysis is done by calculating the mean relative error $E_{m}$ and root-mean-square percent error $E_{r}$, which are defined as

$$
\begin{gathered}
E_{m}=\frac{1}{n} \sum_{j=1}^{n}\left(\frac{\mathrm{TR}_{j}-\mathrm{TM}_{j}}{\mathrm{TM}_{j}}\right) \times 100 \%, \\
E_{r}=\frac{\sqrt{(1 / n) \sum_{j=1}^{n}\left(\mathrm{TR}_{j}-\mathrm{TM}_{j}\right)^{2}}}{\mathrm{TM}_{\text {mean }}} \times 100 \%,
\end{gathered}
$$

where $n$ is the amount of calculating points of the measure area and $\mathrm{TM}_{\text {mean }}$ is the mean temperature of the temperature distribution model, while $\mathrm{TR}_{j}$ and $\mathrm{TM}_{j}$ are the temperatures of reconstructed one and its model of the point $\left(x_{j}, y_{j}\right)$, respectively.

Detecting hotspots is an important issue in microwave field, as hotspots always lead to exceptional and dangerous cases. Thus, positioning ability becomes a vital evaluation indicator of quality of reconstruction performance. Here, the hotspot positioning analysis is done by calculating hotspot positioning error $P_{E}$ and hotspot temperature error $E_{\mathrm{Th}}$, which are defined as

$$
\begin{gathered}
P_{E}=\frac{\sqrt{\left(x_{o}-x_{r o}\right)^{2}-\left(y_{o}-y_{r o}\right)^{2}}}{d} \times 100 \%, \\
E_{\mathrm{Th}}=\left|\frac{\mathrm{TM}_{h}-\mathrm{TR}_{h}}{\mathrm{TM}_{h}}\right| \times 100 \%,
\end{gathered}
$$

where $\left(x_{o}, y_{o}\right)$ and $\mathrm{TM}_{h}$ are the position coordinates and temperature of the hotspot of the temperature distribution model, while $\left(x_{r o}, y_{r o}\right)$ and $\mathrm{TR}_{h}$ are the position coordinates and temperature of the hotspot of the corresponding reconstructed one.
Furthermore, to demonstrate whether the real-time capability of temperature distribution reconstruction using ultrasonic thermometry based on the presented algorithm meets the demand or not, mean reconstruction time of the four kinds of temperature distribution models is calculated. The simulation experiments of each temperature distribution model are repeatedly done 1000 times.

4.2. Simulation Experiment Results Analysis. Figures of results can be a direct-viewing reflection of the reconstruction performance. Here, spatial display and isothermal contour display of temperature distribution models and corresponding reconstructed ones both without and with noise are given, which are shown in Figures 5, 6, 7, and 8.

From the profiles of Figures 5-8, we can intuitively see that all the reconstructed temperature distributions conform well to the model ones, though the reconstruction results of experiments under noises are relatively poor compared to that free from noises.

Quantitative analysis has more powerful persuasiveness with the accurate data of the simulation experiments. Tables 1 and 2 are the overall temperature error analysis results of the simulation experiments of the four temperature distribution models, without noise and with noise, respectively. Tables 3 and 4 are the hotspot positioning analysis results of the simulation experiments of the four temperature distribution models, without noise and with noise, respectively. Mean reconstruction time of these four temperature distribution models is calculated and shown in Table 5.

Obviously, these deviations of temperature distribution reconstruction using ultrasonic thermometry based on our presented algorithm, which are displayed in Tables 1-4, are all small and within the ranges that can be accepted. Moreover, all the reconstruction time of the simulation experiments of the four kinds of temperature distribution models is about $0.18 \mathrm{~s}$ that is far less than $1 \mathrm{~s}$, as displayed in Table 5. It is clear that the real-time capability of temperature distribution reconstruction using ultrasonic thermometry based on the presented algorithm is well enough.

It is concluded from both qualitative analysis and quantitative analysis that temperature distribution reconstruction using ultrasonic thermometry based on our presented 

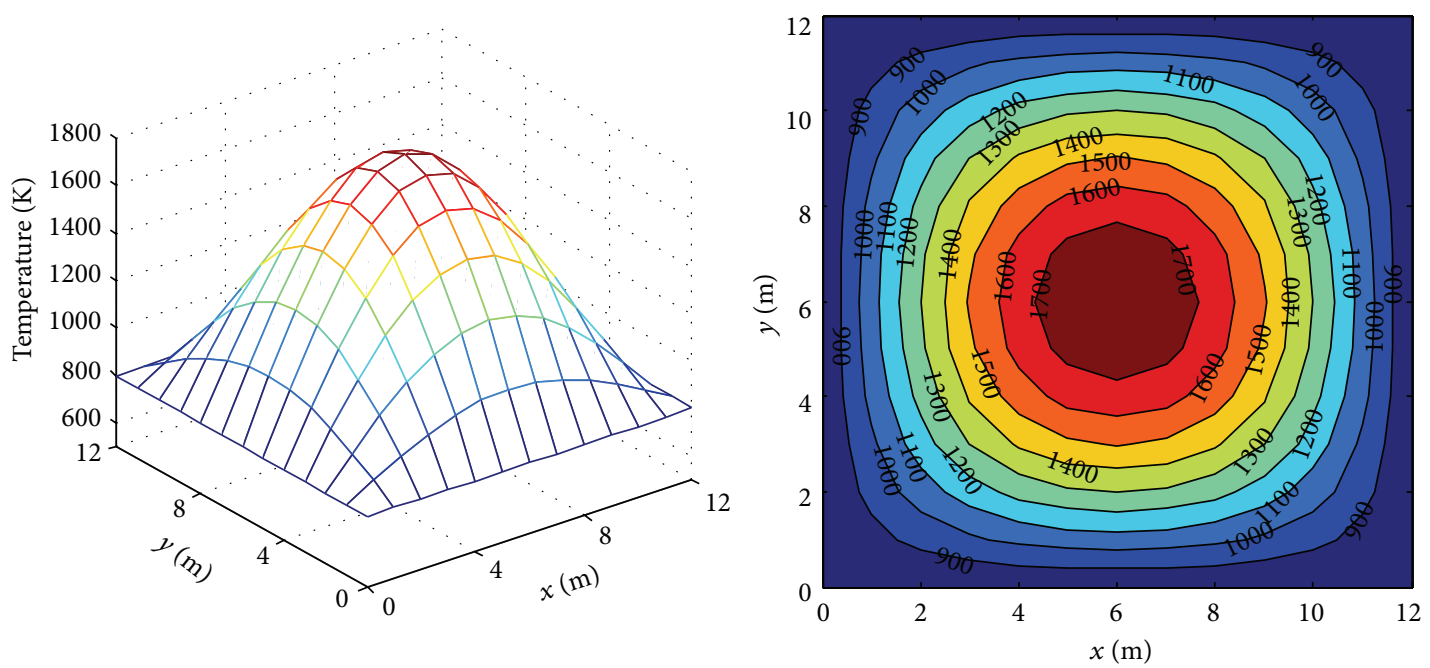

(a) Model
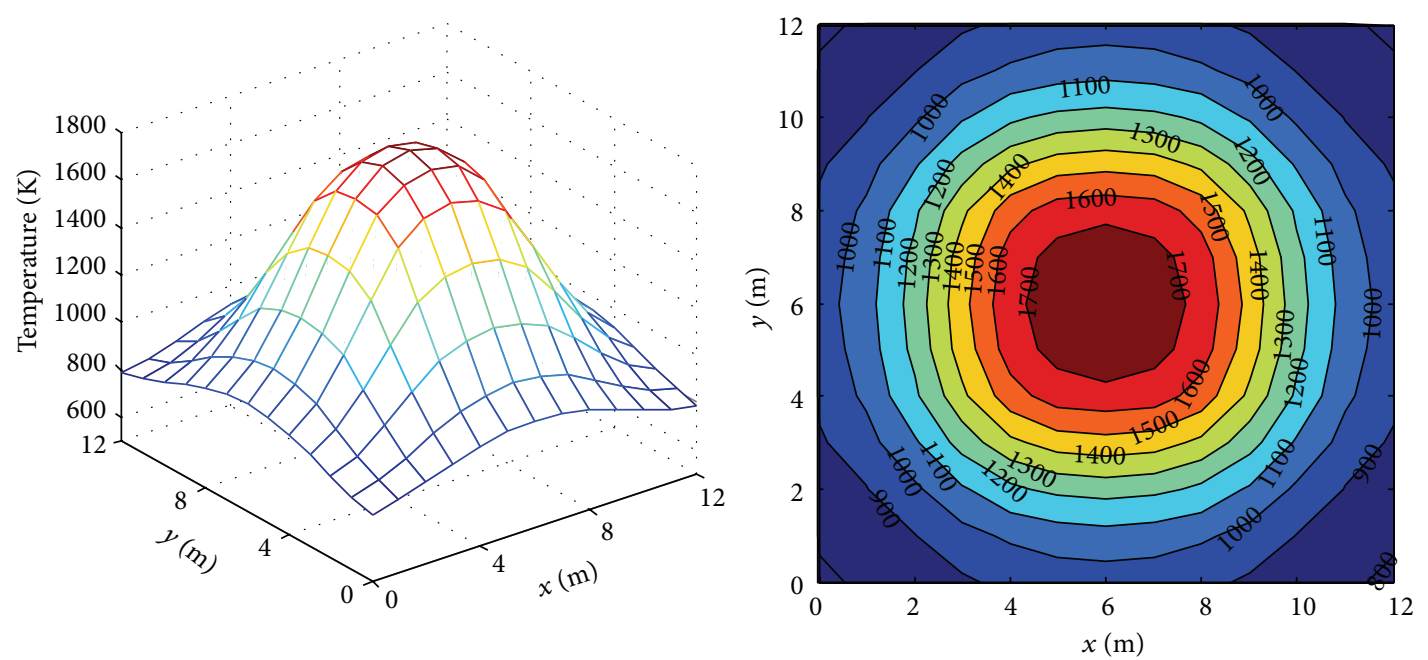

(b) Reconstructed one without noise
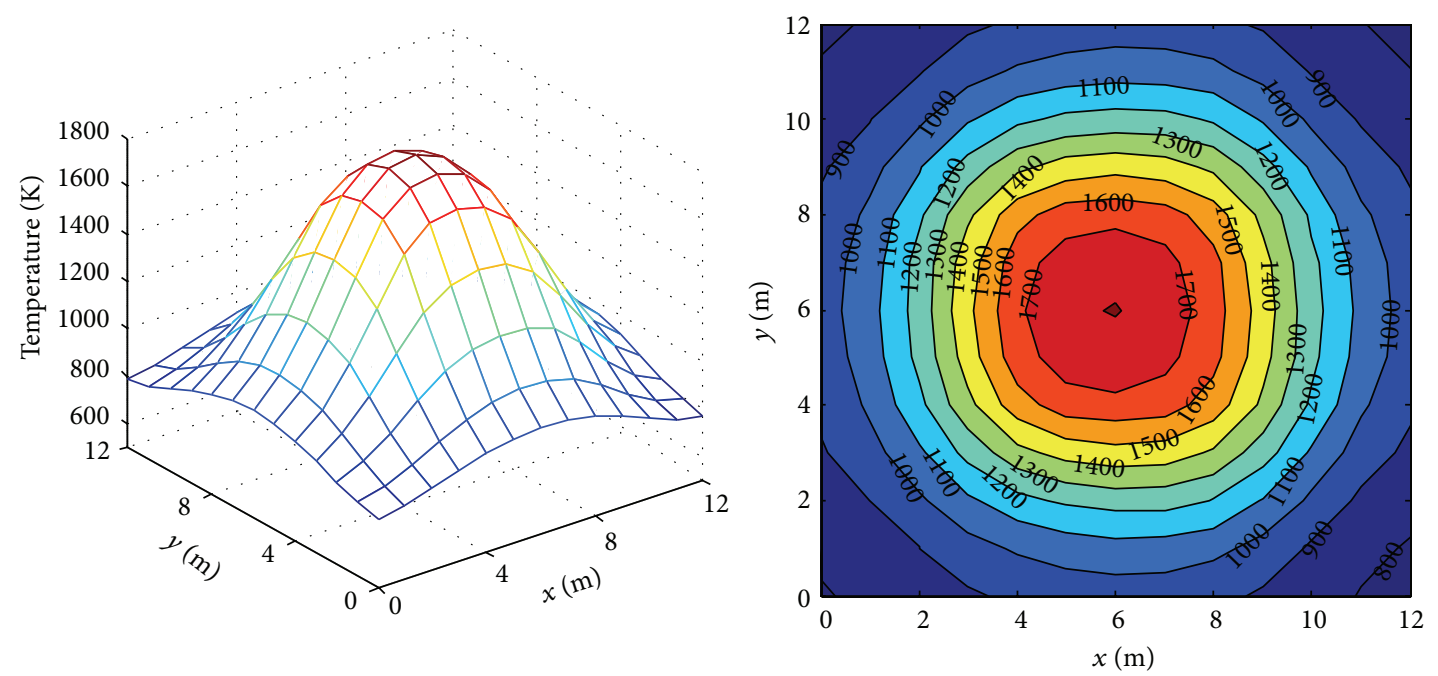

(c) Reconstructed one with Gaussian noise

FIGURE 5: The comparison of experiment results of one-peak symmetrical temperature distribution. 

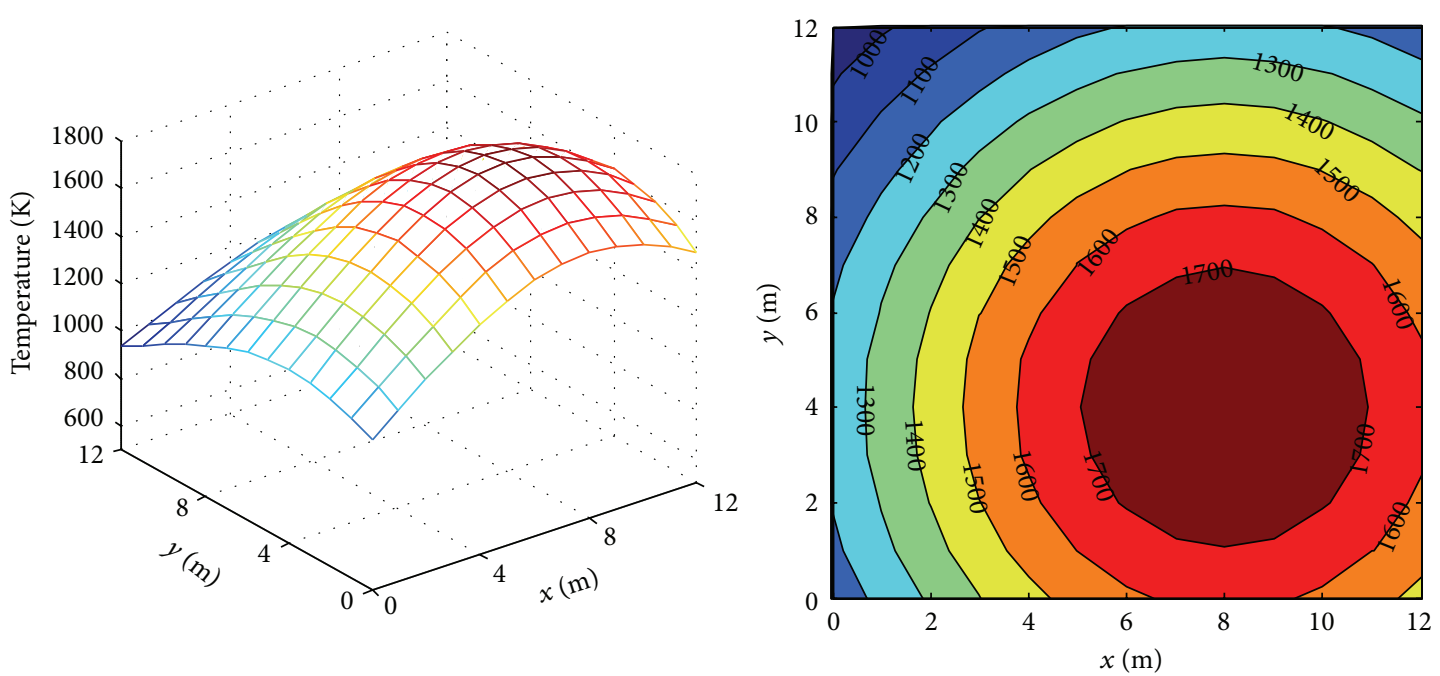

(a) Model
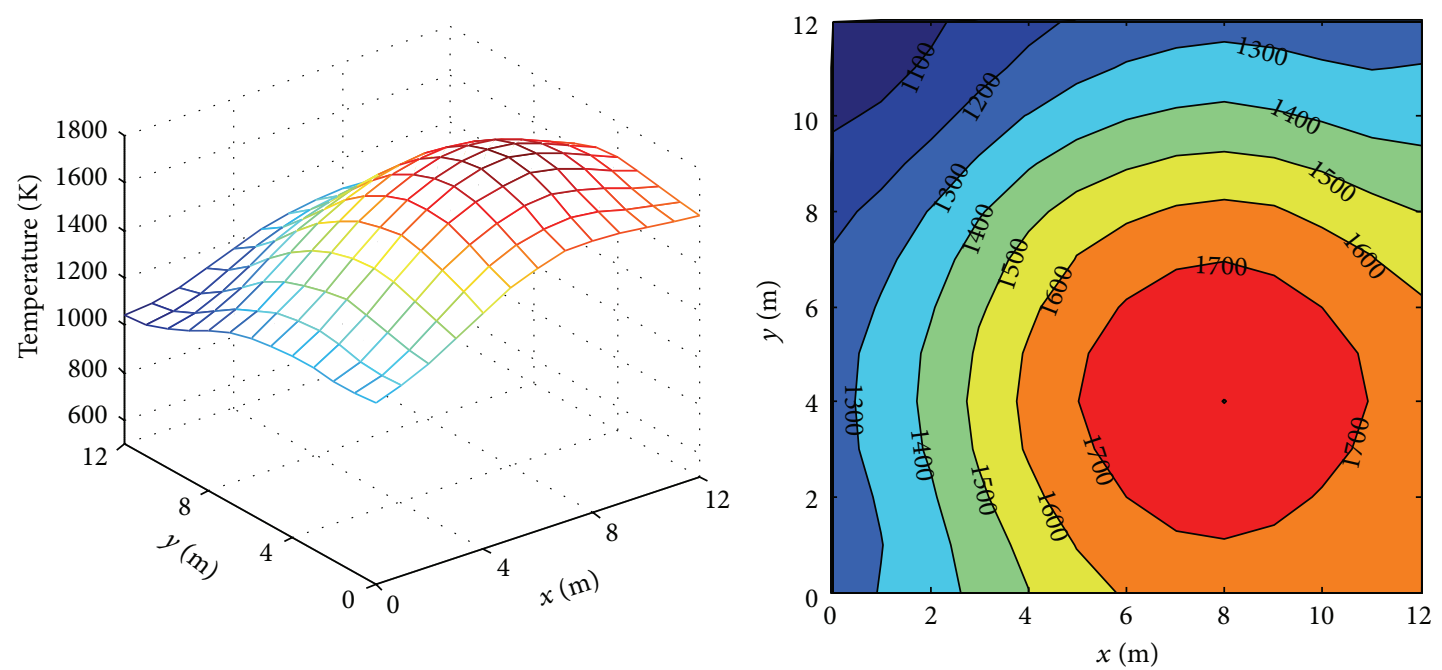

(b) Reconstructed one without noise
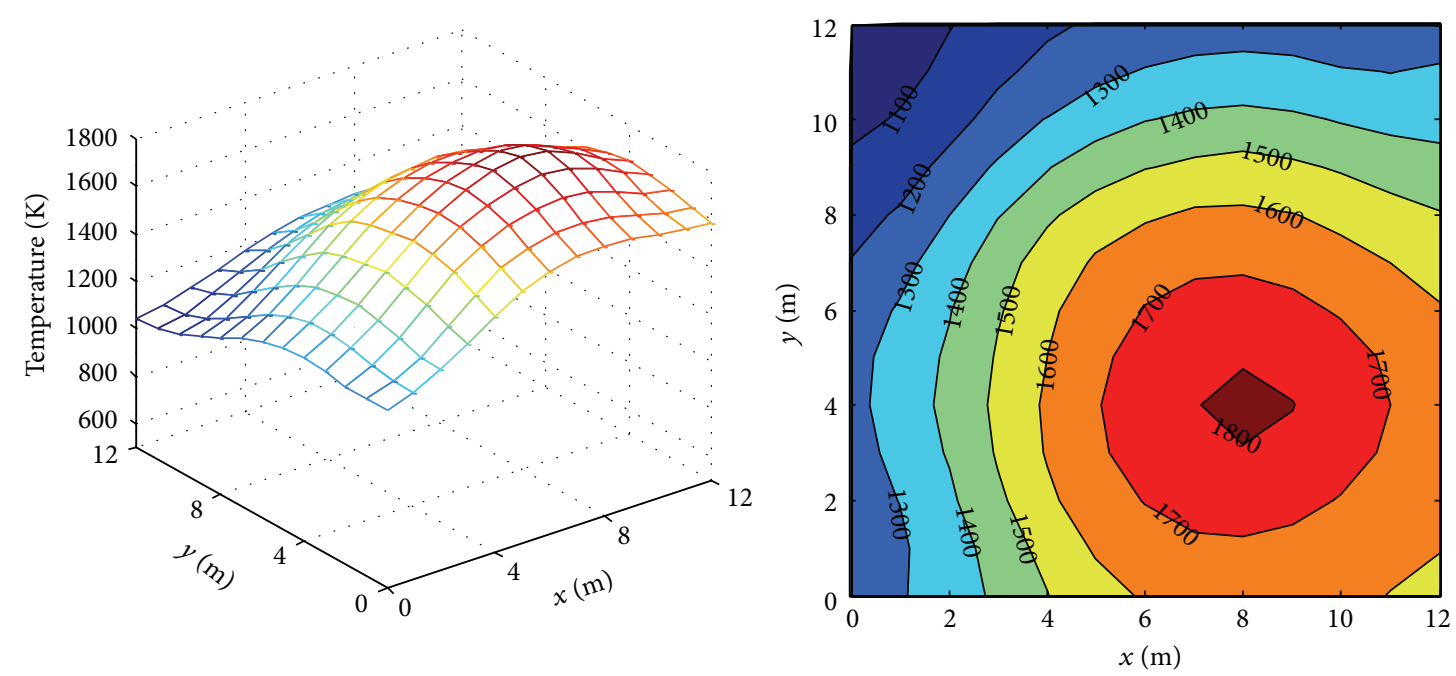

(c) Reconstructed one with Gaussian noise

FIGURE 6: The comparison of experiment results of one-peak asymmetrical temperature distribution. 

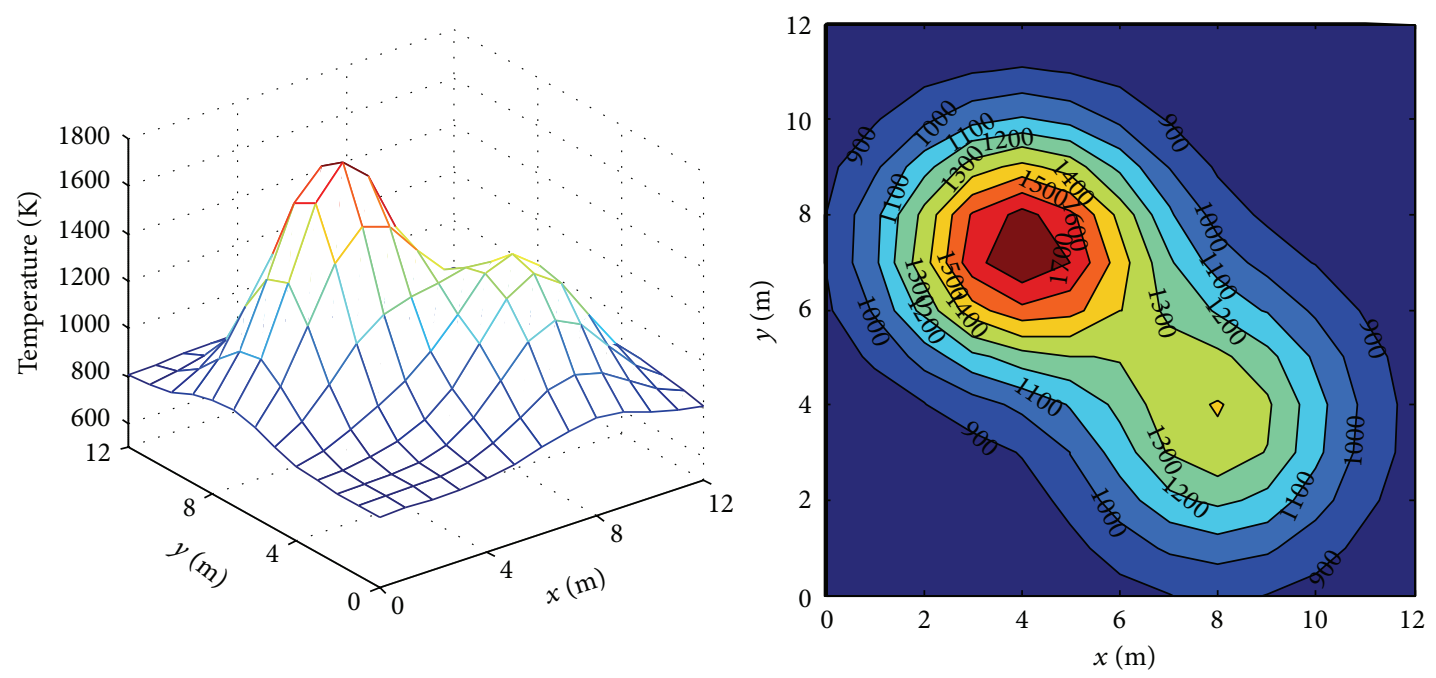

(a) Model
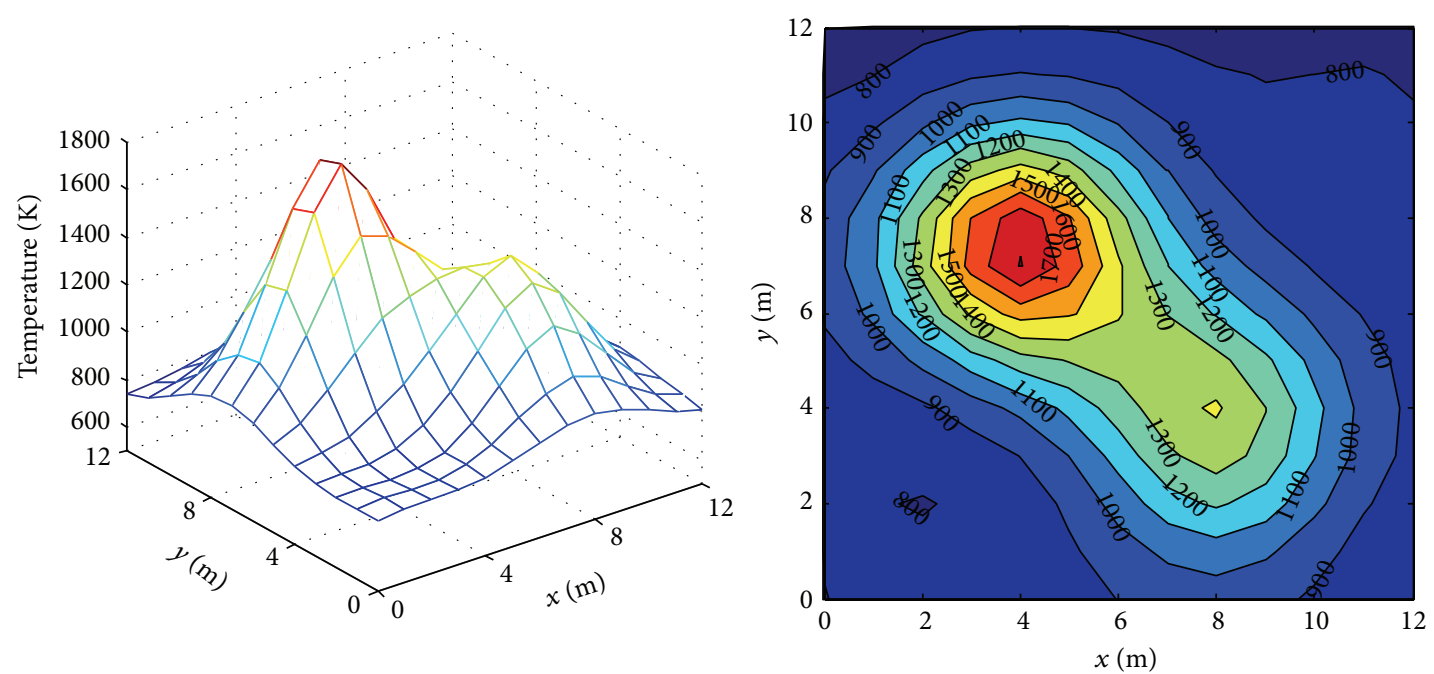

(b) Reconstructed one without noise
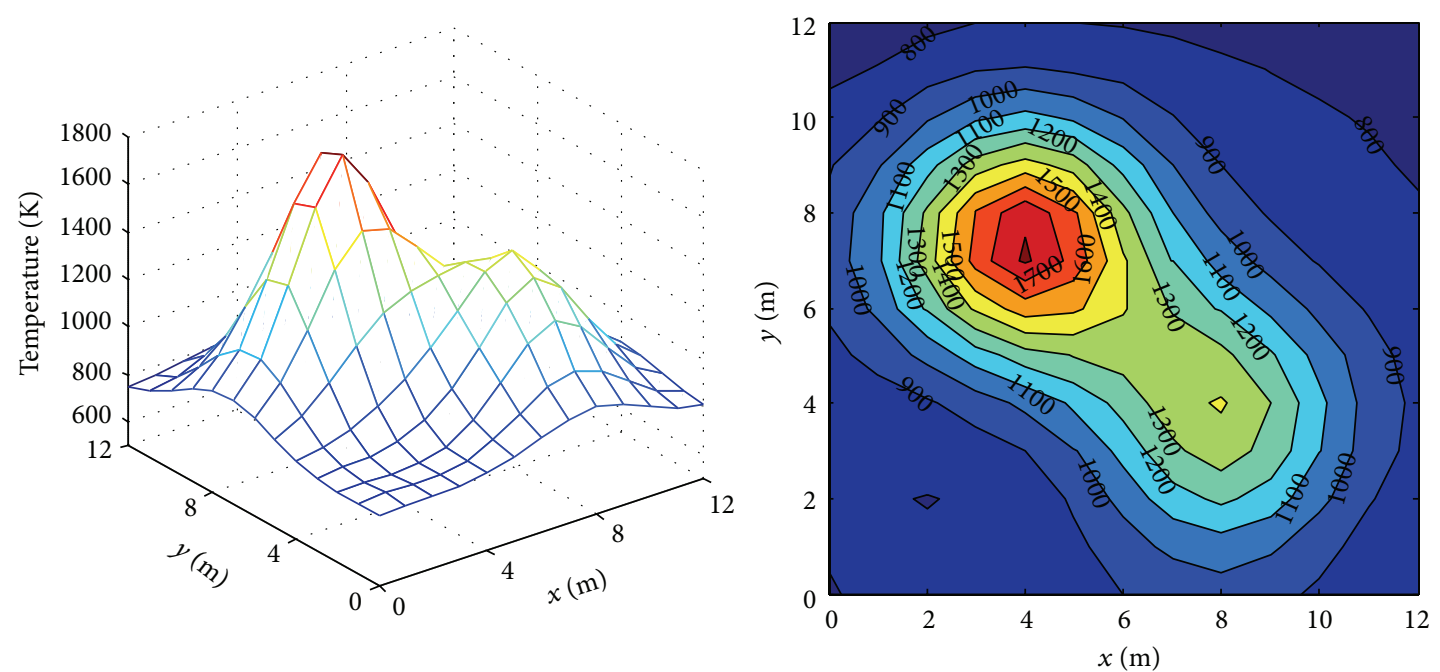

(c) Reconstructed one with Gaussian noise

FIGURE 7: The comparison of experiment results of two-peak asymmetrical temperature distribution. 

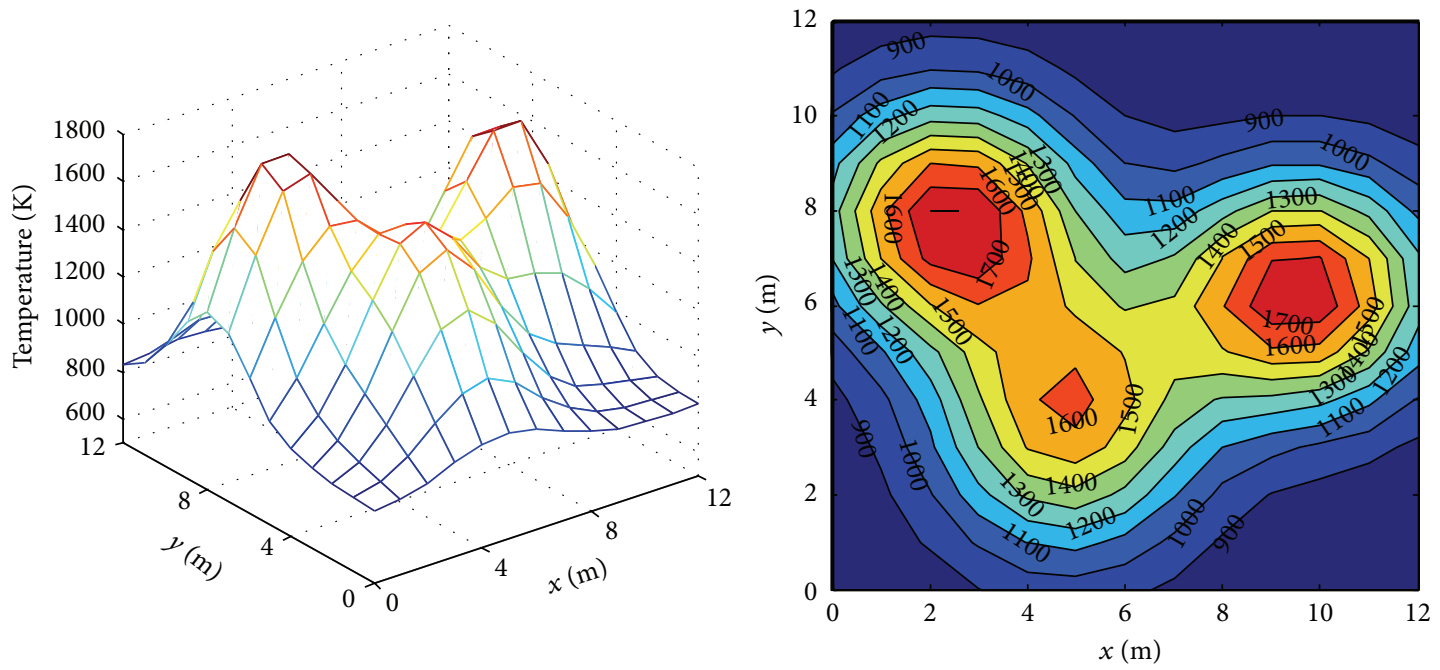

(a) Model
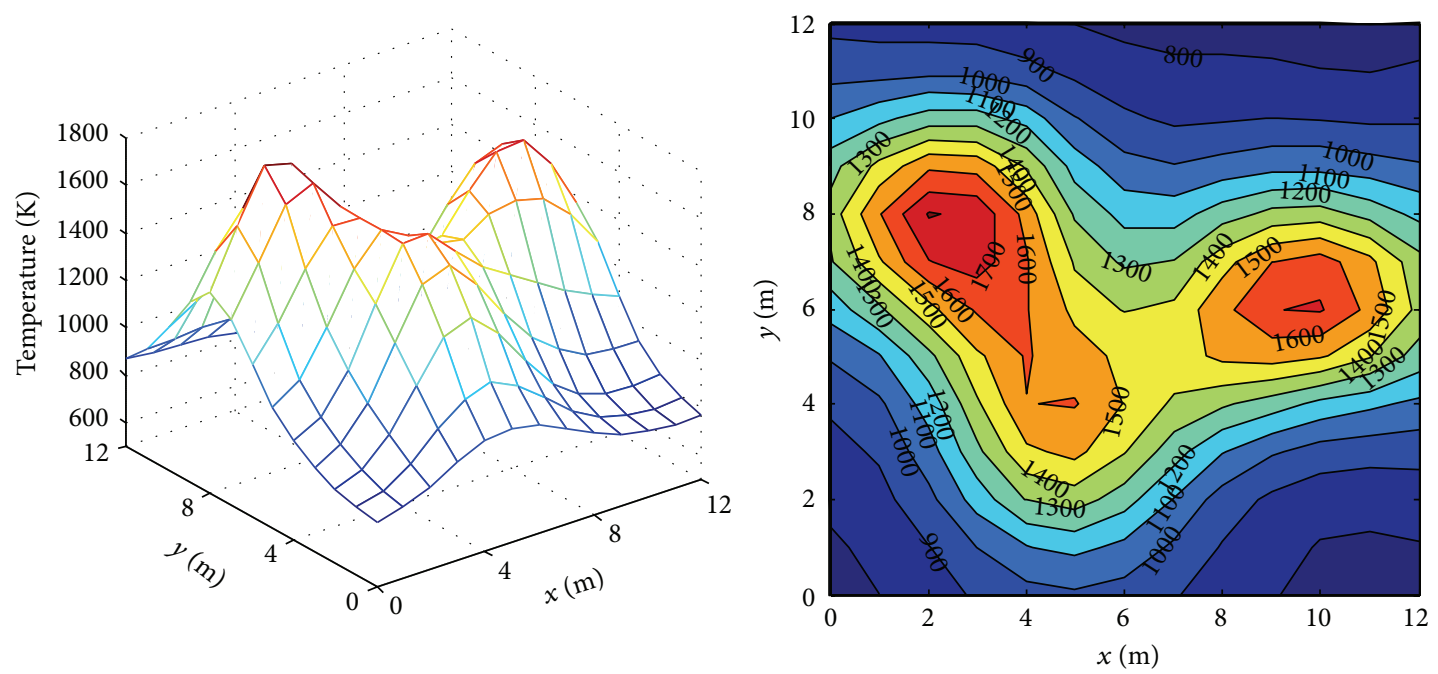

(b) Reconstructed one without noise

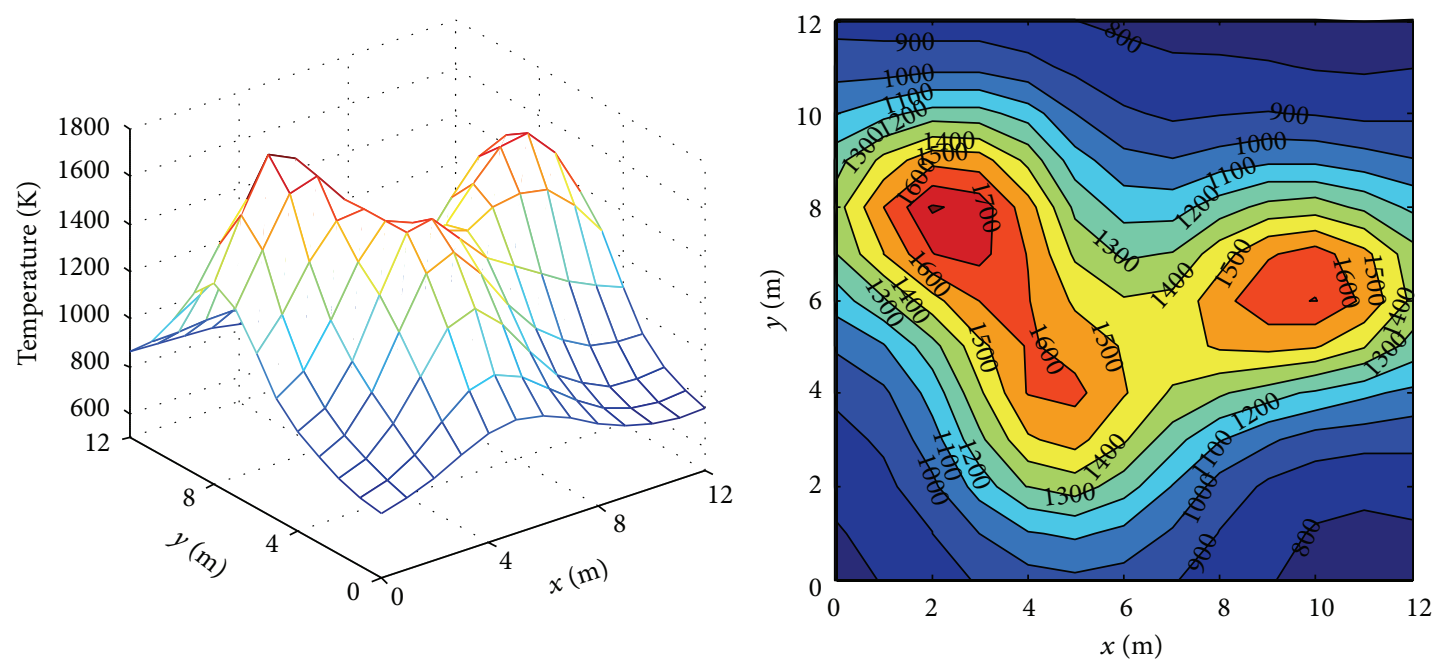

(c) Reconstructed one with Gaussian noise

FIgURE 8: The comparison of experiment results of three-peak asymmetrical temperature distribution. 
TABLE 3: The hotspot positioning analysis (without noise).

\begin{tabular}{lcc}
\hline & Hotspot positioning error $P_{E}$ & Hotspot temperature error $E_{\mathrm{Th}}$ \\
\hline One-peak symmetrical temperature distribution & 0 & $0.281 \%$ \\
One-peak asymmetrical temperature distribution & $2.357 \%$ & $0.065 \%$ \\
Two-peak asymmetrical temperature distribution & $1.863 \%, 2.500 \%$ & $0.064 \%, 0.851 \%$ \\
Three-peak asymmetrical temperature distribution & $4.488 \%, 2.635 \%, 2.357 \%$ & $0.595 \%, 1.785 \%, 3.876 \%$ \\
\hline
\end{tabular}

TABLE 4: The hotspot positioning analysis (with Gaussian noise).

\begin{tabular}{lcr}
\hline & Hotspot positioning error $P_{E}$ & Hotspot temperature error $E_{\text {Th }}$ \\
\hline One-peak symmetrical temperature distribution & $1.454 \%$ & $0.265 \%$ \\
One-peak asymmetrical temperature distribution & $1.964 \%$ & $0.710 \%$ \\
Two-peak asymmetrical temperature distribution & $1.508 \%, 2.500 \%$ & $0.668 \%, 1.023 \%$ \\
Three-peak asymmetrical temperature distribution & $4.488 \%, 3.005 \%, 2.517 \%$ & $0.557 \%, 1.799 \%, 3.942 \%$ \\
\hline
\end{tabular}

TABLE 5: Mean reconstruction time of four temperature distribution models.

\begin{tabular}{lc}
\hline & $\begin{array}{c}\text { Mean reconstruction } \\
\text { time }(\mathrm{s})\end{array}$ \\
\hline $\begin{array}{l}\text { One-peak symmetrical temperature } \\
\text { distribution }\end{array}$ & 0.180826 \\
$\begin{array}{l}\text { One-peak asymmetrical temperature } \\
\text { distribution }\end{array}$ & 0.179738 \\
$\begin{array}{l}\text { Two-peak asymmetrical temperature } \\
\text { distribution }\end{array}$ & 0.180238 \\
$\begin{array}{l}\text { Three-peak asymmetrical temperature } \\
\text { distribution }\end{array}$ & 0.181556 \\
\hline
\end{tabular}

algorithm has high quality performance with admirable feasibility, effectiveness, robustness, and real-time capability and may be used to online monitor the transient temperature distribution in microwave field.

\section{Conclusions and Future Research}

Information about temperature distribution is complex but of critical importance for the control of various microwave applications. In this paper, a practical alternative of temperature distribution monitoring using ultrasonic thermometry in microwave field is investigated. Firstly, detailed description of principle of ultrasonic thermometry is given. And then, typical reconstruction algorithm of temperature distribution based on Markov radial basis function approximation and singular values decomposition is presented. After that, simulation experiments under different conditions are carried out to study the reconstruction performance.

On the basis of the principle and experiment results, we have the following conclusions.

(1) Ultrasonic thermometry can be a noncontact or nonintrusive tool for monitoring temperature distribution in microwave field based on the relationship between the velocity of ultrasound and the properties of the medium which the ultrasound travels through.
(2) The reconstruction algorithm presented based on Markov radial basis function approximation and singular values decomposition is an efficient reconstruction algorithm with high quality reconstruction performance and can detect hotspots accurately.

(3) The reconstruction algorithm presented based on Markov radial basis function approximation and singular values decomposition has well real-time reconstruction capacity, as the reconstruction time calculated is about $0.18 \mathrm{~s}$ which is far less than $1 \mathrm{~s}$.

As the experiments in this paper are conducted in the simulation environment, further validation should be completed by actual experimental setup in a real environment. Different from the simulation experiments, temperature distributions can be constructed using the burners or heating rods to produce high temperature peaks or hot spots. The ultrasonic transducers installed around the measure area of the constructed temperature distribution can be controlled to radiate out and detect ultrasonic signal; then travel time of the paths will be gained and sent to a computer to reconstruct the temperature distribution on the MATLAB platform. Limited by actual experimental conditions, the temperature distributions constructed in real environment may be different from the models artificially created in MATLAB environment more or less. In order to obtain reference data, temperature measurement techniques used for single point can be applied to gain the temperature array of the constructed temperature distribution. For example, array of thermocouples may be interspersed in the constructed temperature distribution to measure the temperatures of the positions in which they are located. Thus, reconstruction performance in real environment can be verified by the contrast between the temperature data of the same point array gained from our presented algorithm and the practical temperature measurement techniques, respectively.

\section{Conflict of Interests}

The authors declare that there is no conflict of interests regarding the publication of this paper. 


\section{Acknowledgment}

This research work is supported by Major State Basic Research Development Program (973 Program Grant no. 2013CB328903).

\section{References}

[1] S. Mutyala, C. Fairbridge, J. R. J. Paré, J. M. R. Bélanger, S. $\mathrm{Ng}$, and R. Hawkins, "Microwave applications to oil sands and petroleum: a review," Fuel Processing Technology, vol. 91, no. 2, pp. 127-135, 2010.

[2] S. Chandrasekaran, S. Ramanathan, and T. Basak, "Microwave material processing-a review," AIChE Journal, vol. 58, no. 2, pp. 330-363, 2012.

[3] A. Motevali, S. Minaei, and M. H. Khoshtagaza, "Evaluation of energy consumption in different drying methods," Energy Conversion and Management, vol. 52, no. 2, pp. 1192-1199, 2011.

[4] M. Nüchter, B. Ondruschka, W. Bonrath, and A. Gum, "Microwave assisted synthesis- a critical technology overview," Green Chemistry, vol. 6, no. 3, pp. 128-141, 2004.

[5] D. A. Jones, T. P. Lelyveld, S. D. Mavrofidis, S. W. Kingman, and N. J. Miles, "Microwave heating applications in environmental engineering - a review," Resources, Conservation and Recycling, vol. 34, no. 2, pp. 75-90, 2002.

[6] I. Manco, L. Giordani, V. Vaccari, and M. Oddone, "Microwave technology for the biodiesel production: analytical assessments," Fuel, vol. 95, no. 1, pp. 108-112, 2012.

[7] J. Y. Jeon and H. Y. Kim, "Microwave irradiation effect on diffusion of organic molecules in polymer," European Polymer Journal, vol. 36, no. 5, pp. 895-899, 2000.

[8] K. I. Rybakov, E. A. Olevsky, and E. V. Krikun, "Microwave sintering: fundamentals and modeling," Journal of the American Ceramic Society, vol. 96, no. 4, pp. 1003-1020, 2013.

[9] L. Wu, H. C. Zhu, and K. M. Huang, "Thermal analysis on the process of microwave-assisted biodiesel production," Bioresource Technology, vol. 133, pp. 279-284, 2013.

[10] S. V. Egorov, K. I. Rybakov, V. E. Semenov et al., "Role of convective heat removal and electromagnetic field structure in the microwave heating of materials," Journal of Materials Science, vol. 42, no. 6, pp. 2097-2104, 2007.

[11] D. Stuerga and P. Gaillard, "Microwave heating as a new way to induce localized enhancements of reaction rate," Tetrahedron, vol. 52, no. 15, pp. 5505-5510, 1996.

[12] S. Moriwaki, M. MacHida, H. Tatsumoto, M. Kuga, and T. Ogura, "A study on thermal runaway of poly(vinyl chloride) by microwave irradiation," Journal of Analytical and Applied Pyrolysis, vol. 76, no. 1-2, pp. 238-242, 2006.

[13] C. A. Vriezinga, S. Sánchez-Pedreno, and J. Grasman, "Thermal runaway in microwave heating: a mathematical analysis," Applied Mathematical Modelling, vol. 26, no. 11, pp. 1029-1038, 2002.

[14] G. S. J. Sturm, M. D. Verweij, T. van Gerven, A. I. Stankiewicz, and G. D. Stefanidis, "On the effect of resonant microwave fields on temperature distribution in time and space," International Journal of Heat and Mass Transfer, vol. 55, no. 13-14, pp. 38003811, 2012.

[15] K. Knoerzer, M. Regier, and H. Schubert, "Microwave heating: a new approach of simulation and validation," Chemical Engineering and Technology, vol. 29, no. 7, pp. 796-801, 2006.
[16] K. M. Huang, H. C. Zhu, and L. Wu, "Temperature cycle measurement for effective permittivity of biodiesel reaction," Bioresource Technology, vol. 131, pp. 541-544, 2013.

[17] K. Berdel, J. G. Rivas, P. H. Bolívar, P. de Maagt, and H. Kurz, "Temperature dependence of the permittivity and loss tangent of high-permittivity materials at terahertz frequencies," IEEE Transactions on Microwave Theory and Techniques, vol. 53, no. 4, pp. 1266-1271, 2005.

[18] G. Kychakoff, A. F. Hollingshead, and S. P. Boyd, "Use of acoustic temperature measurements in the cement manufacturing pyroprocess," in Proceedings of the IEEE Cement Industry Technical Conference Record, pp. 23-33, May 2005.

[19] A. Kosugi, I. Ihara, and I. Matsuya, "Accuracy evaluation of surface temperature profiling by a laser ultrasonic method," Japanese Journal of Applied Physics, vol. 51, no. 7, Article ID 07GB01, 2012.

[20] W.-Y. Tsai, H.-C. Chen, and T.-L. Liao, "High accuracy ultrasonic air temperature measurement using multi-frequency continuous wave," Sensors and Actuators A: Physical, vol. 132, no. 2, pp. 526-532, 2006.

[21] T. Durka, G. D. Stefanidis, T. V. Gerven, and A. Stankiewicz, "On the accuracy and reproducibility of fiber optic (FO) and infrared (IR) temperature measurements of solid materials in microwave applications," Measurement Science \& Technology, vol. 21, no. 4, Article ID 045108, 2010.

[22] E. Belotserkovsky, O. Shenfeld, and A. Katzir, "Infrared fiberoptic temperature control of the heating process in a microwave oven," IEEE Transactions on Microwave Theory and Techniques, vol. 42, no. 5, pp. 901-903, 1994.

[23] K. Srinivasan, T. Sundararajan, and S. Narayanan, "Acoustic pyrometry in flames," Measurement, vol. 46, no. 1, pp. 315-323, 2013.

[24] T. A. Hanson, N. Yilmaz, P. Drozda, W. Gill, T. J. Miller, and A. B. Donaldson, "Acoustic pyrometry using an off-the-shelf range finding system," Journal of Fire Sciences, vol. 26, no. 4, pp. 287308, 2008.

[25] M. Bramanti, E. A. Salerno, A. Tonazzini, S. Pasini, and A. Gray, "An acoustic pyrometer system for tomographic thermal imaging in power plant boilers," IEEE Transactions on Instrumentation and Measurement, vol. 45, no. 1, pp. 159-167, 1996.

[26] R. J. Steriti and M. A. Fiddy, "Regularized image reconstruction using SVD and a neural network method for matrix inversion," IEEE Transactions on Signal Processing, vol. 41, no. 10, pp. 30743077, 1993.

[27] D. Modgil, B. E. Treeby, and P. J. la Riviere, "Photoacoustic image reconstruction in an attenuating medium using singularvalue decomposition," Journal of Biomedical Optics, vol. 17, no. 6, Article ID 061204, 2012.

[28] V. V. Selivanov and R. Lecomte, "Fast PET image reconstruction based on SVD decomposition of the system matrix," IEEE Transactions on Nuclear Science, vol. 48, no. 3, pp. 761-767, 2001. 


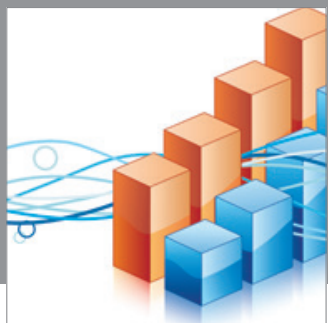

Advances in

Operations Research

mansans

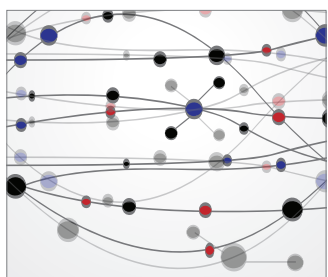

The Scientific World Journal
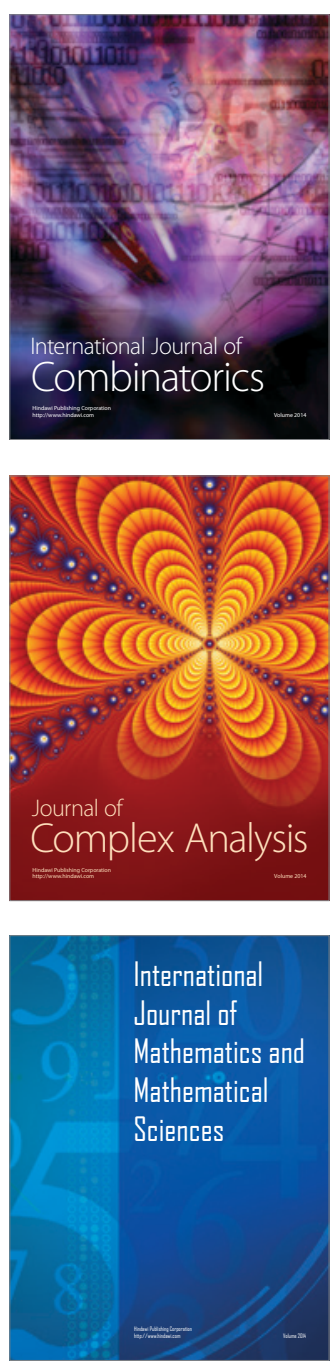
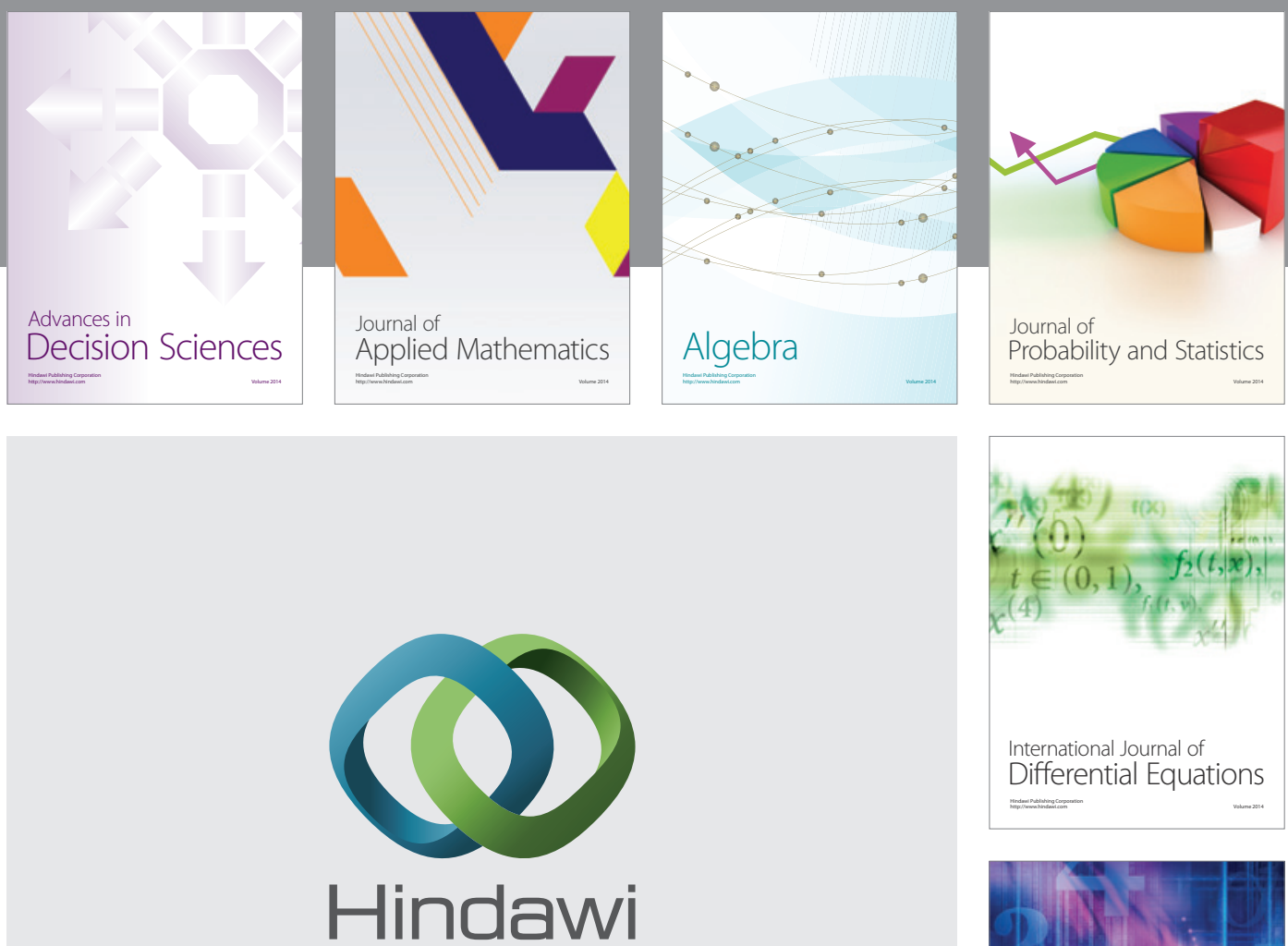

Submit your manuscripts at http://www.hindawi.com
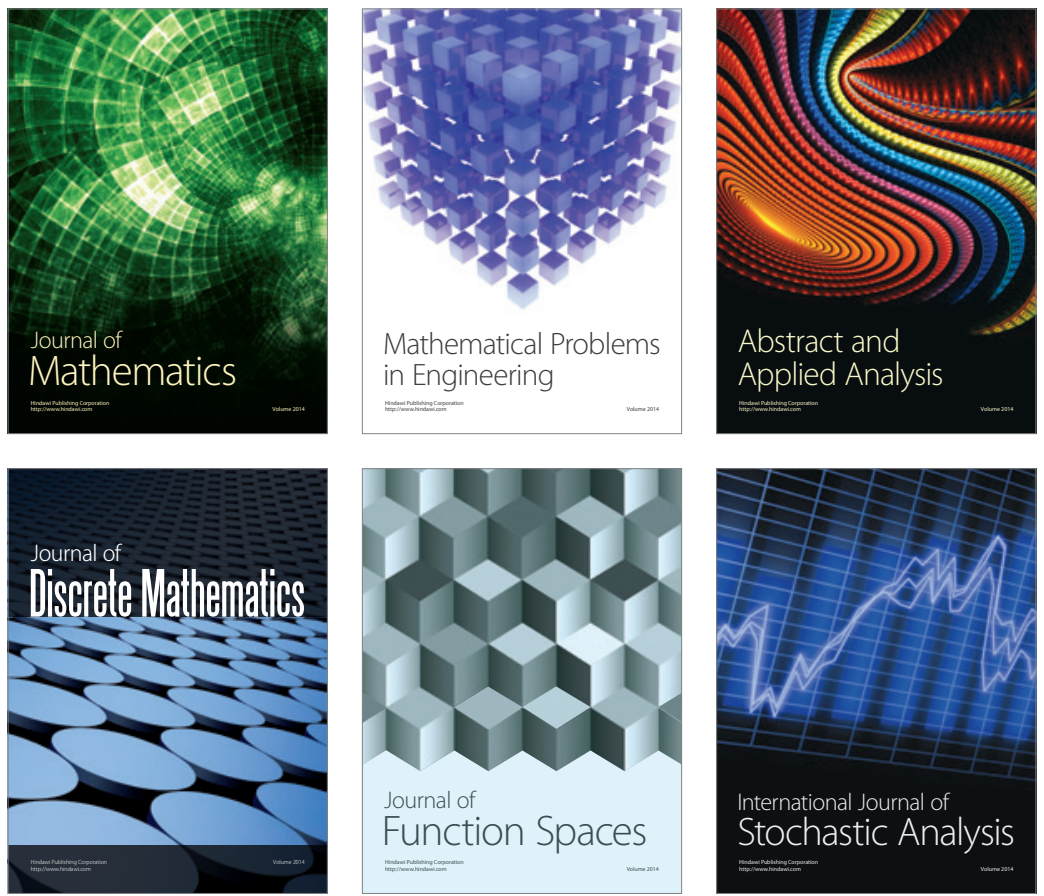

Journal of

Function Spaces

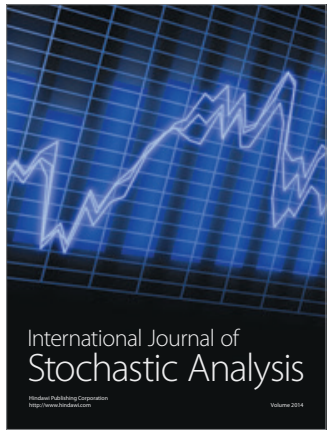

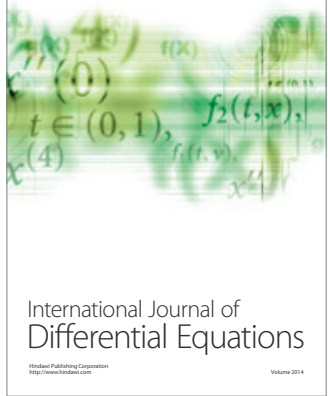
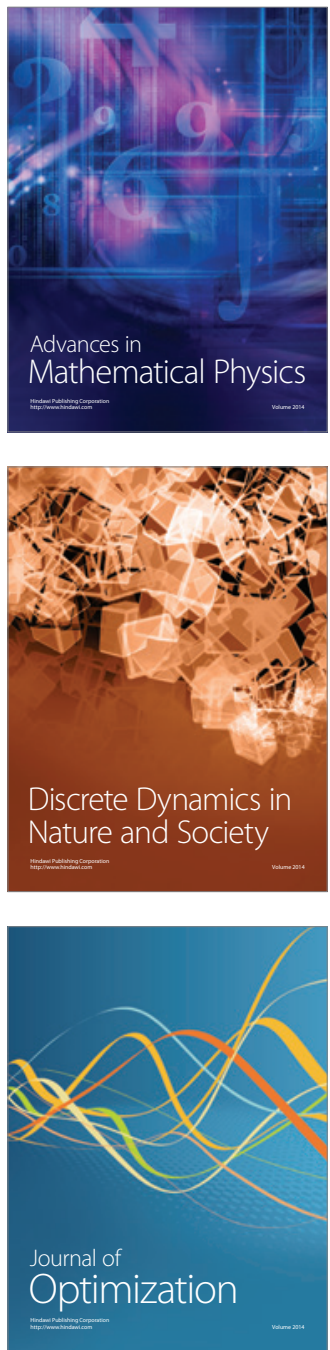